\title{
Knowledge, Awareness, and Behavior of Medical and Dental Interns toward Human Immunodeficiency Virus/ Acquired Immunodeficiency Syndrome Patients in Three Colleges of Madhya Pradesh, India: A Cross-sectional Survey
}

\author{
${ }^{1}$ Kodangala P Ashok, ${ }^{2}$ Anjani Tripathi, ${ }^{3}$ Sahana Ashok
}

\begin{abstract}
Introduction: India is one of the countries where a large population is affected by human immunodeficiency virus (HIV). It destroys the specific immune system cells called CD4 (cluster of differentiation 4) cells leading to an advanced condition called acquired immunodeficiency syndrome (AIDS). It is one of the life-threatening conditions. It is an infectious disease that can affect caregivers as well. These patients need to be treated with compassion and caution. The doctors need to be aware of the various types of infection, their symptoms, and precautions while treating them. Hence, there is a need to evaluate awareness and behavior among the medical and dental interns while dealing with patients suffering from HIV infection/AIDS patients.
\end{abstract}

Materials and methods: The present cross-sectional survey explored knowledge, awareness, and behavior of medical and dental interns toward HIV-infected/AIDS patients at People's University, Bhopal, India. A questionnaire consisting of 25 questions was given to the interns. A total of 350 interns were included in the study; 250 interns were from People's College of Dental Sciences \& Research Centre and People's Dental Academy, Bhopal, India, while 100 interns were from People's College of Medical Sciences, Bhopal, India. The collected data were analyzed and subjected to relevant statistics.

Results: Out of 350 questionnaires received, 250 were answered by dental interns and 100 were answered by medical interns. The mean and standard deviation (SD) of percentage knowledge score for dental interns was $76.57 \pm 17.67$ and for medical interns, it was $83.05 \pm 11.04$. Median of percentage knowledge score was 84.80 and 86.00 for dental and medical interns respectively. Mann-Whitney U-test showed no significant difference between dental and medical interns for percentage knowledge scores $(p>0.05)$. The awareness score between the dental and medical interns showed that dental interns (82.34) showed slightly better awareness to the HIV

\footnotetext{
${ }^{1}$ Professor, ${ }^{2}$ Postgraduate Student, ${ }^{3}$ Senior Lecturer

${ }^{1,2}$ Department of Periodontics, People's College of Dental Sciences \& Research Centre, Bhopal, Madhya Pradesh, India

${ }^{3}$ Department of Oral Pathology, People's Dental Academy, Bhopal Madhya Pradesh, India

Corresponding Author: Kodangala P Ashok, Professor Department of Periodontics, People's College of Dental Sciences \& Research Centre, Bhopal, Madhya Pradesh, India Phone: +91992959750, e-mail: drashokkp@gmail.com
}

patients than medical interns (81.00), although it was not statistically significant. When the behavior scores were compared between the dental and medical interns, the medical interns showed a higher score (85.4) compared with the dental interns (76.80), which was statistically significant $(p<0.05)$.

Conclusion: The cross-sectional survey showed that medical students had better knowledge but dental interns had better awareness, though both were statistically not significant.

The medical interns had better behavior score than dental interns, which was statistically significant.

Keywords: Awareness, Behavior, Control, Education, Human immunodeficiency virus/acquired immunodeficiency syndrome, Knowledge.

How to cite this article: Ashok KP, Tripathi A, Ashok S. Knowledge, Awareness, and Behavior of Medical and Dental Interns toward Human Immunodeficiency Virus/Acquired Immunodeficiency Syndrome Patients in Three Colleges of Madhya Pradesh, India: A Cross-sectional Survey. J Health Sci Res 2017;8(2):66-71.

\section{Source of support: Nil}

Conflict of interest: None

\section{INTRODUCTION}

In today's era, HIV/AIDS cases are increasing at tremendous pace, accounting to many deaths in the developing countries like India; AIDS is the end stage of HIV infection, which is caused by the HIV virus and is a lifethreatening condition. There are two types of patients with HIV infection. ${ }^{1}$ A section of patients are unaware of their own infection, but the second section of patients hide their disease status because of social stigma. Health care workers (HCWs) including dentists are under risk of contamination as strict protocols are not followed. The HCWs should incorporate a healthy attitude toward persons suffering with HIV infection/AIDS. Therefore, a thorough knowledge, awareness, and behavior play an important role toward the medical and dental care of HIV-infected individuals.

In general, HIV is very rarely transmitted during oral health care treatment. ${ }^{2}$ Detection of HIV infection is important as lesions are usually seen orally during the 
first stage of the disease. ${ }^{3}$ The oral manifestations occur as an outcome of the compromised immune system. The medical and dental care of HIV infection/AIDS patients requires proper understanding of the disease, modes of transmission, oral manifestations, etc. The goal is to improve the quality of patient's life by analyzing, identifying, and improving their health. ${ }^{4}$ The present study aims to assess the knowledge, awareness, and behavior of medical and dental interns toward HIV infections/ AIDS patient encountered during routine medical and dental care.

\section{MATERIALS AND METHODS}

A total of 350 interns participated in the study, out of which 250 dental interns were from People's College of Dental Sciences and Research Centre and People's Dental Academy and 100 medical interns from People's College of Medical Sciences, Bhopal, India. A questionnaire containing 25 multiple choice questions, in which the students had to choose only one answer from the

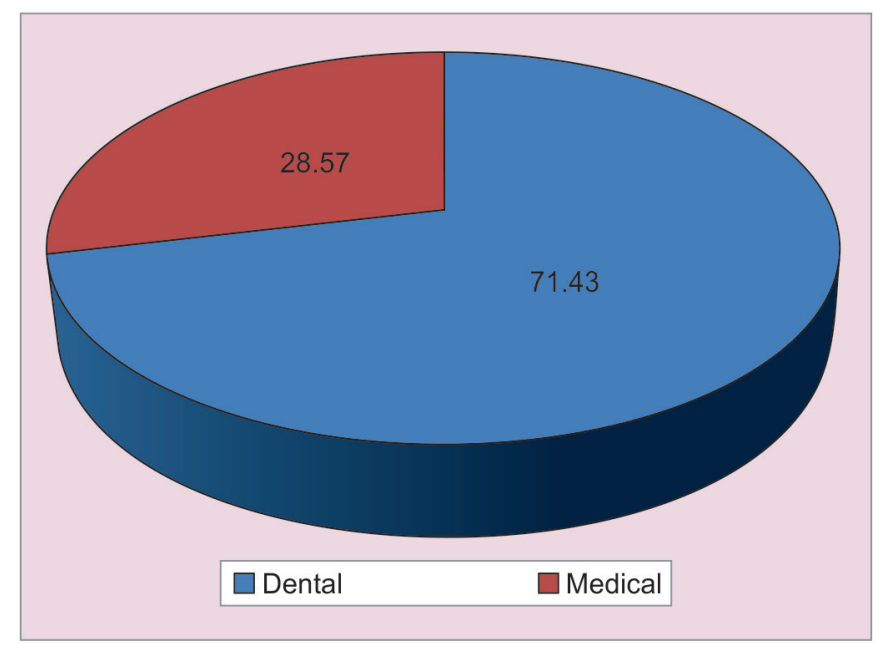

Graph 1: Distribution of study subjects provided list of options, was given to the participants. This questionnaire included questions pertaining to knowledge, awareness, and behavior while handling HIV infection/AIDS patients during routine medical/dental care. The students received an explanation of how to fill the questionnaire.

\section{RESULTS}

Graph 1 indicates the distribution of number of dental (71.43) and medical interns (28.57) in the study. Few questions pertaining to knowledge are described in Graph 2 with response given by medical and dental interns (Table 1).

The knowledge score percentage was 83.05 for medical interns and 76.57 for dental interns as indicated in Graph 3 (Table 2). A total of 206 (82.4\%) dental interns and 91 (91\%) medical interns responded that syphilis, HIV, and Hepatitis B virus are the occupational diseases of dentists. Fisher's exact test showed significantly higher number of medical interns correctly responded than dental interns $(\mathrm{p}<0.05) ; 112(44.8 \%)$ dental interns and 65 (65\%) medical interns responded that not everyone who is exposed to HIV gets infected. Fisher's exact test showed significantly higher number of medical interns correctly responded than dental interns ( $\mathrm{p}<0.01) .224(89.60 \%)$ dental interns and 74 (74\%) medical interns responded that key reason for death of a HIV infected patient is infections occurring due to breakdown of immune system. Fisher's exact test showed significantly higher number of dental interns correctly responded than medical interns $(\mathrm{p}<0.001)$ (Table 1 and Graphs 1 and 2).

In the study, 138 (55.20\%) dental interns responded that there is adequate awareness created in our society about HIV and AIDS. However, 85 (85\%) medical interns did not agree with this (Tables 3 and 4). Fisher's exact test showed significant difference between dental and

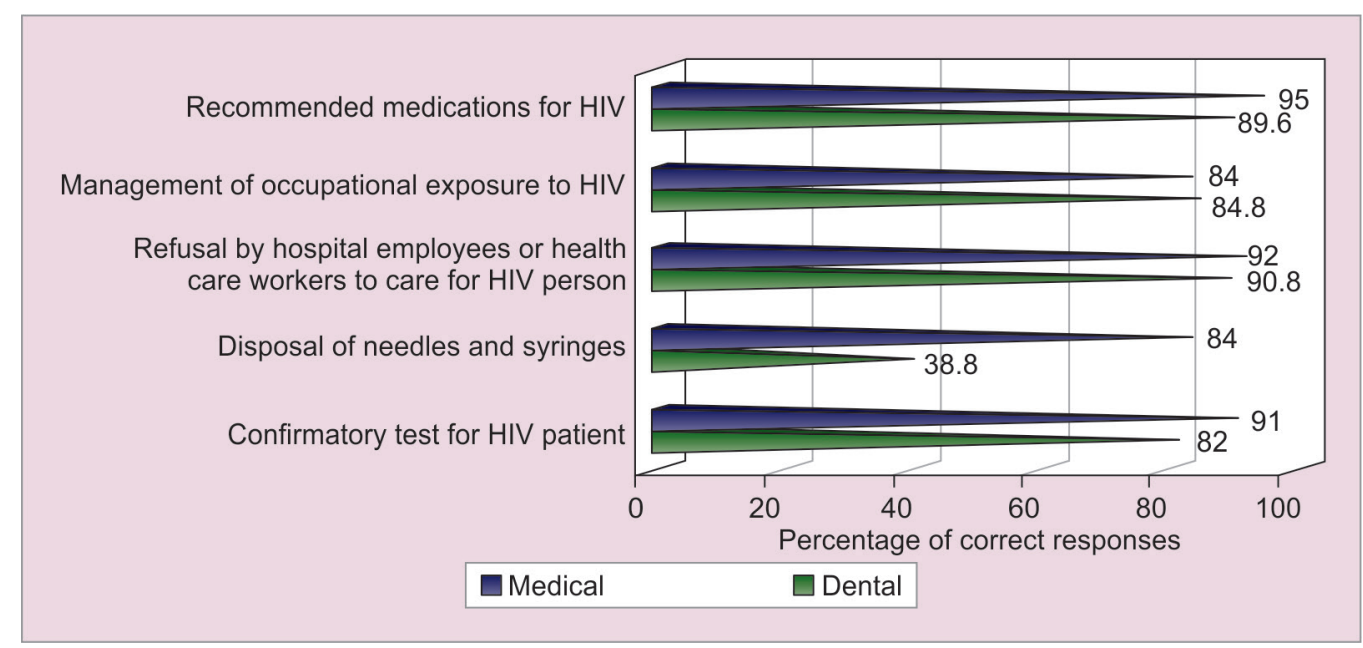

Graph 2: Percentage of correct responses by dental $(n=250)$ and medical $(n=100)$ interns for knowledge questions 
Table 1: Responses by dental $(n=250)$ and medical $(n=100)$ interns for knowledge-based questions

\begin{tabular}{lllll}
\hline & \multicolumn{3}{c}{ Correct responses } \\
\cline { 2 - 4 } Question & Dental interns $n(\%)$ & Medical interns $n(\%)$ & Total $n(\%)$ & Fisher's exact $p$-value \\
\hline Difference between HIV infection and AIDS & $220(88.00)$ & $90(90.00)$ & $310(88.57)$ & $0.711(>0.05), \mathrm{NS}$ \\
Occupational diseases of dentists & $206(82.40)$ & $91(91.00)$ & $297(84.86)$ & $0.048(<0.05), \mathrm{S}$ \\
Everyone who is exposed to HIV gets infected & $112(44.80)$ & $65(65.00)$ & $177(50.57)$ & $0.001(<0.01), \mathrm{S}$ \\
World AIDS day & $194(77.60)$ & $81(81.00)$ & $275(78.57)$ & $0.565(>0.05), \mathrm{NS}$ \\
Confirmatory test for HIV patient & $205(82.00)$ & $91(91.00)$ & $296(84.57)$ & $0.035(<0.05), \mathrm{S}$ \\
Maximum transmission of HIV & $180(72.00)$ & $81(81.00)$ & $261(74.57)$ & $0.103(>0.05), \mathrm{NS}$ \\
Disposal of needles and syringes & $97(38.80)$ & $84(84.00)$ & $181(51.71)$ & $0(<0.001), \mathrm{S}$ \\
Medical care providers for HIV patients & $214(85.60)$ & $86(86.00)$ & $300(85.71)$ & $1.000(>0.05), \mathrm{NS}$ \\
Preference of disinfection over sterilization & $95(38.00)$ & $62(62.00)$ & $157(44.86)$ & $0(<0.001), \mathrm{S}$ \\
Lesion strongly associated with HIV infection & $215(86.00)$ & $87(87.00)$ & $302(86.29)$ & $0.865(>0.05), \mathrm{NS}$ \\
Management of occupational exposure to HIV & $212(84.80)$ & $84(84.00)$ & $296(84.57)$ & $0.871(>0.05), \mathrm{NS}$ \\
Symptoms of AIDS patient & $217(86.80)$ & $86(86.00)$ & $303(86.57)$ & $0.863(>0.05), \mathrm{NS}$ \\
Recommended medications for HIV patients & $224(89.60)$ & $95(95.00)$ & $319(91.14)$ & $0.144(>0.05), \mathrm{NS}$ \\
\hline
\end{tabular}

\section{S: Significant; NS: Nonsignificant}

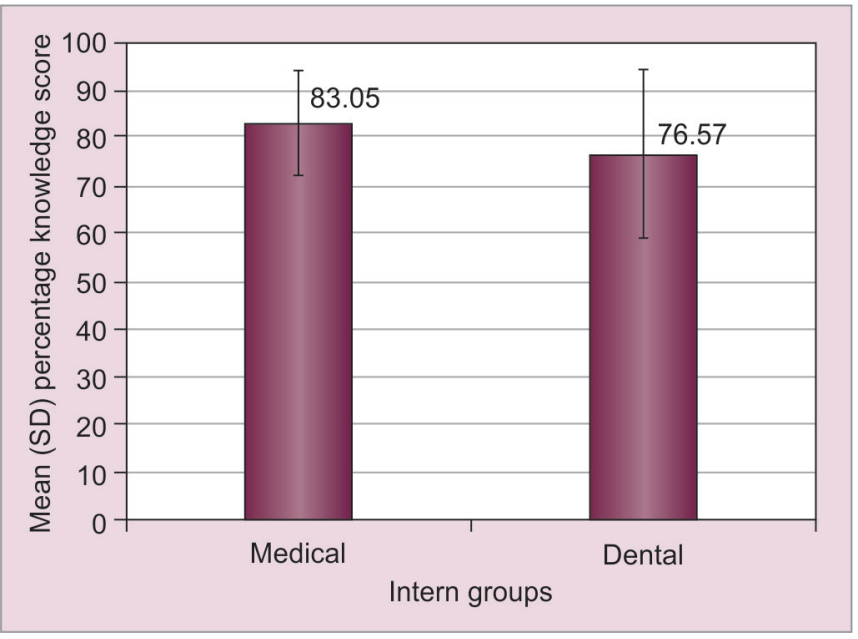

Graph 3: Mean and SD of percentage knowledge score in dental and medical interns

medical interns for the responses ( $\mathrm{p}<0.001) ; 145(58.00 \%)$ dental interns and $48(48.00 \%)$ medical interns responded that there is adequate awareness among medical and dental interns in treating HIV patients. Fisher's exact
Table 2: Comparison of percentage knowledge score between dental and medical interns

\begin{tabular}{llll}
\hline & \multicolumn{3}{c}{ Percentage knowledge score } \\
\cline { 2 - 4 } Intern groups & Mean $\pm S D$ & Median & Min-Max \\
\hline Dental & $76.57 \pm 17.67$ & 84.80 & $38.00-93.20$ \\
Medical & $83.05 \pm 11.04$ & 86.00 & $52.00-95.00$ \\
\hline Mann-Whitney & U-test value $=163.00, \mathrm{p}=0.148(>0.05)$, \\
nonsignificant & &
\end{tabular}

test showed no significant difference between dental and medical interns for the responses ( $\mathrm{p}>0.05) ; 233$ $(93.20 \%)$ dental interns and $95(95.00 \%)$ medical interns responded that they follow strict sterilization protocol while handling AIDS patients. Fisher's exact test showed no significant difference between dental and medical interns for the responses ( $p>0.05) ; 230(92.00 \%)$ dental interns and $90(90.00 \%)$ medical interns responded that their institution follows strict sterilization protocol while treating AIDS patients. Fisher's exact test showed no significant difference between dental and medical interns for the responses $(p>0.05)$ (Graph 4).

Table 3: Responses by dental $(n=250)$ and medical $(n=100)$ interns for awareness-based questions

\begin{tabular}{llllll}
\hline Emotional counseling is helpful & Yes & $198(79.20)$ & $90(90.00)$ & $288(82.29)$ & $0.020(<0.05)$, S \\
& No & $52(20.80)$ & $10(10.00)$ & $62(17.71)$ & \\
Occupational exposure risk reduction for health & Yes & $219(87.60)$ & $84(84.00)$ & $303(86.57)$ & $0.388(>0.05)$, NS \\
care workers & No & $31(12.40)$ & $16(16.00)$ & $47(13.43)$ & \\
Institution follows strict protocol while treating & Yes & $230(92.00)$ & $90(90.00)$ & $320(91.43)$ & $0.532(>0.05)$, NS \\
AIDS patients & No & $20(8.00)$ & $10(10.00)$ & $30(8.57)$ & \\
Features of suspected AIDS patient in outpatient & Yes & $233(93.20)$ & $91(91.00)$ & $324(92.57)$ & $0.501(>0.05)$, NS \\
department & No & $17(6.80)$ & $9(9.00)$ & $26(7.43)$ & \\
First test for a suspected AIDS patient before any & Yes & $123(49.20)$ & $52(52.00)$ & $175(50.00)$ & $0.638(>0.05)$, NS \\
treatment & No & $27(50.80)$ & $48(48.00)$ & $175(50.00)$ & \\
Medical care providers for HIV patients & Yes & $214(85.60)$ & $86(86.00)$ & $300(85.71)$ & $1.000(>0.05)$, NS \\
& No & $36(14.40)$ & $14(14.00)$ & $50(14.29)$ & \\
Key reason for death of a HIV infected patient & Yes & $224(89.60)$ & $74(74.00)$ & $298(85.14)$ & $0(<0.001), \mathrm{S}$ \\
& No & $26(10.40)$ & $26(26.00)$ & $52(14.86)$ &
\end{tabular}

S: Significant; NS: Nonsignificant 
Table 4: Comparison of percentage awareness score between dental and medical interns

\begin{tabular}{lll}
\hline Intern groups & Mean $\pm S D$ & $\begin{array}{l}\text { Wilcoxon signed rank } \\
\text { test } p \text {-value }\end{array}$ \\
\hline Dental & $82.34 \pm 15.33$ & 0.612 \\
Medical & $81.00 \pm 14.05$ & \\
\hline
\end{tabular}

$(>0.05)$ Nonsignificant

\section{Statistical Analysis}

Data were entered in Microsoft Excel 2016. Responses by interns were dichotomized as correct responses and wrong responses. Frequencies, percentages, mean, SD, minimum and maximum values of responses were calculated. To compare responses of dental and medical interns, Fisher's exact test was applied. To calculate knowledge score for 12 knowledge-based questions, correct response was given score 1 , whereas wrong as well as no responses were given score 0 . To facilitate the comparison, scores were converted into percentages. Shapiro-Wilk test showed that percentage knowledge scores did not follow normal distribution. Hence, MannWhitney U-test (nonparametric test) was used for comparison of knowledge scores between dental and medical interns. Wilcoxon signed rank test was used to compare the awareness and behavior scores of dental and medical interns; p-value of $<0.05$ was considered statistically significant. Data analyses were performed using version 21.0 of the Statistical Package for the Social Sciences (IBM Corporation, Armonk, New York, USA).

Mean and SD of percentage knowledge score for dental interns was $76.57 \pm 17.67$ and for medical interns was $83.05 \pm 11.04$ (Table 2). Median of percentage knowledge score was 84.80 and 86.00 for dental and medical interns respectively. The minimum and maximum percentage scores for dental interns were 38.00 to 93.20 and for medical interns were 52.00 to 95.00 (Graph 5).
Mann-Whitney U-test showed no significant difference between dental and medical interns for knowledge scores percentage $(p>0.05)$.

The awareness score between the dental and medical interns showed that dental interns (82.34) showed slightly better awareness to the HIV patients than medical interns (81.00). Wilcoxon signed ranks test was used. It was not statistically significant (Tables 3, 4 and Graph 4).

When the scores were compared between the dental and medical interns, the medical interns showed a higher score (85.4) compared with the dental interns (76.80). Wilcoxon signed rank test was used and was statistically significant (Tables 5, 6 and Graph 5).

\section{DISCUSSION}

Our study about the responsiveness on knowledge, awareness, and behavior about medical and dental care in HIV infection/AIDS patients shows that majority of students have adequate knowledge regarding the modes of transmission and regarding sterilization protocols. Medical interns, when compared with dental interns, did not agree that adequate awareness has been created in our society regarding HIV/AIDS. Mukhopadhyay et al ${ }^{5}$ in a survey on college students observed that $<60 \%$ had satisfactory knowledge on AIDS. Knowledge is an important factor that may be related to behavioral change. ${ }^{4}$ Educational programs designed to increase knowledge will have an effect on behavior and will prevent the spread of HIV infection. ${ }^{6}$ Student's attitude toward HIVinfected individuals is variable. Their attitude can be changed over a period of time by proper education and sensitization about HIV infection/AIDS. Further emphasis needs to be given through workshops and seminars. Anuradha and Lakshmi ${ }^{6}$ in a study showed that, out of total of 144 students from 1st year, i.e., 100 from medical and 44 from dental courses, $93.75 \%$ of students knew that

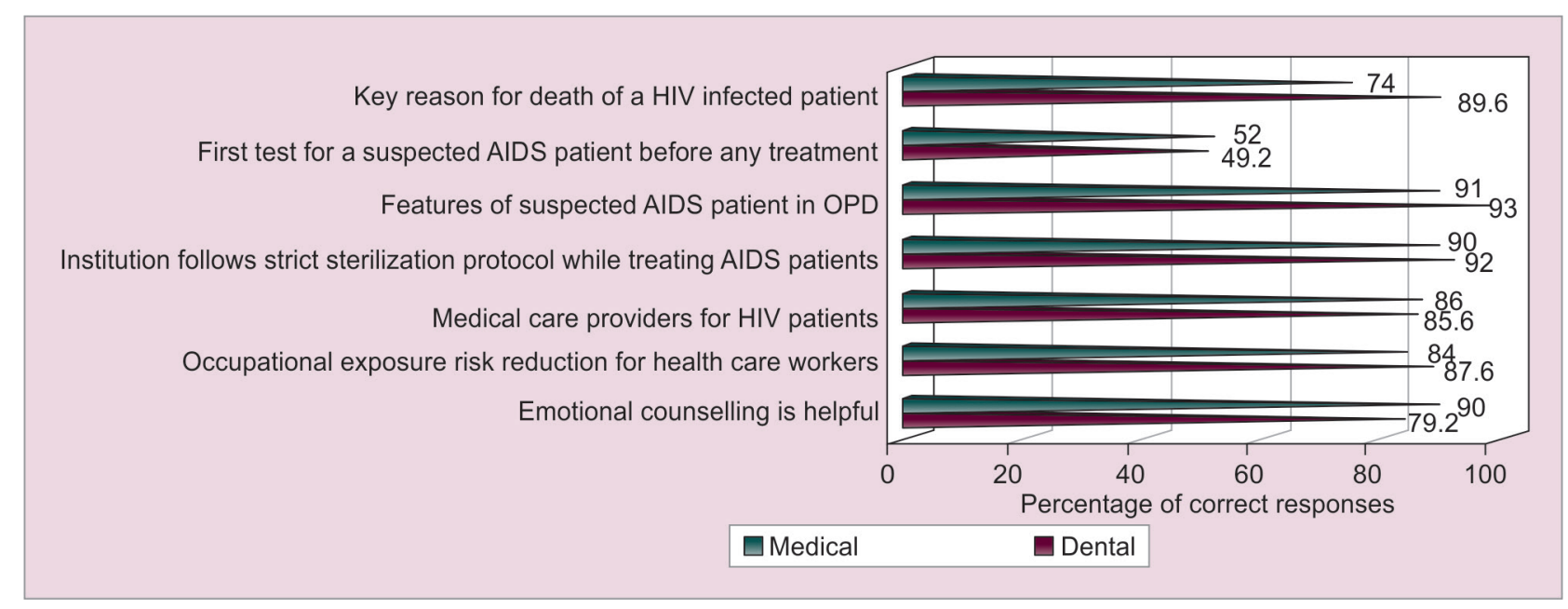

Graph 4: Percentage of correct responses by dental $(n=250)$ and medical $(n=100)$ interns for awareness questions 


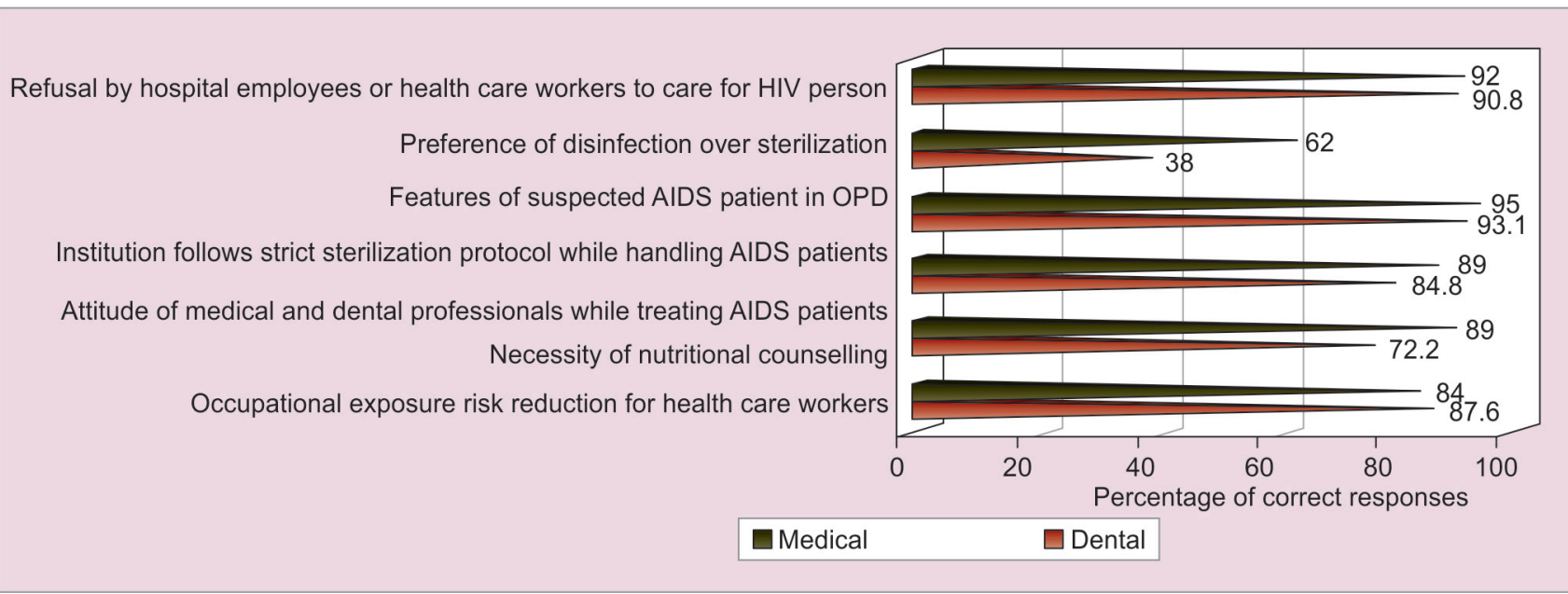

Graph 5: Percentage of correct responses by dental $(n=250)$ and medical $(n=100)$ interns for health behavior questions

Table 5: Responses by dental $(n=250)$ and medical $(n=100)$ interns for behavior-based questions

\begin{tabular}{llllll}
\hline Necessity of nutritional counseling & Yes & $193(77.20)$ & $89(89.00)$ & $282(80.57)$ & $0(<0.001), \mathrm{S}$ \\
& No & $57(22.80)$ & $11(11.00)$ & $68(19.43)$ & \\
Attitude of medical and dental professionals while & Yes & $212(84.80)$ & $89(89.00)$ & $301(86.00)$ & $0.394(>0.05)$, NS \\
treating AIDS patients & No & $38(15.20)$ & $11(11.00)$ & $49(14.0)$ & \\
Following strict sterilization protocol while handling & Yes & $233(93.20)$ & $95(95.00)$ & $328(93.71)$ & $0.632(>0.05)$, NS \\
AIDS patients & No & $17(6.8)$ & $5(5.00)$ & $22(6.29)$ & \\
Refusal by hospital employees or health care & Yes & $227(90.80)$ & $92(92.00)$ & $319(91.14)$ & $0.837(>0.05)$, NS \\
workers to care for HIV person & No & $23(9.20)$ & $8(8.00)$ & $31(8.86)$ & \\
Preference of disinfection over sterilization & Yes & $95(38.00)$ & $62(62.00)$ & $157(44.86)$ & $0(<0.001), \mathrm{S}$ \\
& No & $155(62.00)$ & $38(38.00)$ & $193(55.14)$ &
\end{tabular}

S: Significant; NS: Nonsignificant

it is transmitted through unprotected sex, 91.6\% knew that it may spread by blood transfusion, 77\% knew about vertical transmission from mother to fetus, and $84.7 \%$ had the knowledge about spread through infected IV needle and drug abuse. The majority of students (67.3\%) knew the meaning and abbreviations of HIV/AIDS. A large number of students (93.75\%) were aware of the mode of transmission through unprotected sex. In a study conducted by Albujeer et $\mathrm{al}^{7}$ in 600 students, he found that the knowledge of about half of the medical students (54\%) was at an intermediate level and of $27.1 \%$ students was at a good level; more than half of the dental students $(68.2 \%)$ had an intermediate level and $10.5 \%$ had a good level of knowledge. The level of attitude of medical students was $14.7 \%$ at an intermediate level and of dentistry students was $21.4 \%$ at an intermediate level. Precaution while dealing with HIV infection/AIDS patients is also a matter of concern. In a study carried out by Al-Naimi and Al-Saygh, ${ }^{8}$ it was seen that $90 \%$ of the students were using all three types of barrier techniques.

The awareness score between the dental and medical interns showed that dental interns (82.34) showed slightly better awareness to the HIV patients than medical interns (81.00). This was not statistically significant. This showed that both groups of interns had good awareness regarding the protocols of treatment; 90 (90\%) medical students said that emotional counseling is helpful when compared with 198 (79.2\%) of dental interns. Both groups of interns believed that their institution follows proper sterilization protocols. Dental health staff are exposed to infectious agents during work, especially when proper barrier precautions are not followed. ${ }^{9}$ When the behavior scores were compared between the dental and medical interns, the medical interns showed a higher score (85.4) compared with the dental interns (76.80) (Table 5). This was statistically significant (Tables 5 and 6). Both groups of interns were of the opinion that HIV patients can be refused treatment in certain conditions. More number of medical interns (62\%) preferred disinfection over sterilization than dental interns (38\%). More medical interns said that nutritional counseling is essential compared with the dental interns (Graph 5).

Table 6: Comparison of percentage behavior score between dental and medical interns

\begin{tabular}{lll}
\hline Intern groups & Mean $\pm S D$ & $\begin{array}{l}\text { Wilcoxon signed } \\
\text { ranks test } p \text {-value }\end{array}$ \\
\hline Dental & $76.80 \pm 13.3$ & 0.043 \\
Medical & $85.40 \pm 22.55$ & \\
\hline
\end{tabular}

$(<0.05)$ Significant 
Health care professionals play a crucial role in prevention and control of HIV infection/AIDS, and it is necessary to assess knowledge and behavior of health care professionals for overcoming ignorance and spreading awareness. ${ }^{10}$ Appropriate training can expedite learning of affirmative behaviors and attitudes toward HIV/AIDS patients among medical/dental students. This study necessitates the need for a more thorough assessment of student's knowledge, awareness, and behavior among the student groups.

\section{CONCLUSION}

The cross-sectional survey showed that medical students had better knowledge but dental interns had better awareness, though both were statistically not significant.

The medical interns had better behavior score than dental interns, which was statistically significant.

\section{REFERENCES}

1. ChauhanAS,Hussain MA,PatiS,NallalaS,MishraJ.Knowledge and attitudes related to HIV/AIDS among medical and allied health sciences students. Ind J Comm Health 2011 Dec; 23(2):96-98.

2. Baron S, Poast J, Cloyd MW. Why is HIV rarely transmitted by oral secretions? Saliva can disrupt orally shed, infected leukocytes. Arch Intern Med 1999 Feb;159(3):303-310.
3. Grando LJ, Yurgel LS, Machado DC, Nachman S, Ferguson F, Berentsen B, Fernandes A. The association between oral manifestations and the socioeconomic and cultural characteristics of HIV-infected children in Brazil and in the Unites States of America. Rev Panam Salud Publica 2003 Aug;14(2): 112-118.

4. Rai JJ, Acharya RV, Dave D. Knowledge and awareness among interns and residents about medical law and negligence in a medical college in Vadodara-a questionnaire study. JDMS 2013 Jan-Feb;3(4):32-38.

5. Mukhopadhyay BB, Mukherjee B, Biswas AB. Knowledge and attitude about AIDS among the newly admitted college students in a West Bengal town. AIDS Asia 1996;6:18.

6. Anuradha B, Lakshmi S. Awareness about HIV/AIDS among medical entrants: a questionnaire study. NJBMS 2015 Jul-Sep;6(1):14-17.

7. Albujeer ANH, Shamshiri AR, Taher A. HIV/AIDS awareness among Iraqi medical and dental students. J Int Soc Prev Community Dent 2015 Sep-Oct;5(5):372-375.

8. Al-Naimi RJ, Al-Saygh GD. Knowledge, attitude and health behavior of dental students towards HIV patients. Dent J 2009;9(1)110-119.

9. Shan V, Shethwala ND, Bala DV. Knowledge, attitude and health behavior of dental students towards HIV patients. Healthline 2011;2(1)58-60.

10. Yousuf A, Shah AF, Jan SM, Sidiq M, Baba IA. Awareness of HIV/AIDS infection and ethical concerns amongst dentistry students and auxiliary staff in a hospital setup in Kashmir, India. Int J Community Med Public Health 2016 Oct;3(10):2850-2855. 